\title{
PROPORTIONAL CROSSTALK FOR THE iThemba LABS CLOVER DETECTOR*
}

\author{
S.P. Noncolela ${ }^{a, b}$, T.D. Bucher ${ }^{a, c}$, E.A. LAWRIE ${ }^{a}$, T.R.S. DinOKO ${ }^{a}$

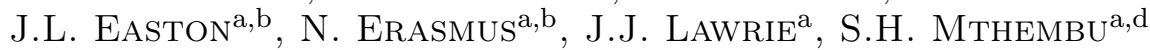

 \\ aiThemba LABS, National Research Foundation \\ P.O. Box 722, 7129 Somerset West, South Africa \\ ${ }^{\mathrm{b}}$ Department of Physics and Astronomy, University of the Western Cape \\ P/B X17, 7535 Bellville, South Africa \\ ${ }^{\mathrm{c}}$ Department of Physics, Stellenbosch University \\ P/B X1, Matieland 7602, South Africa \\ ${ }^{\mathrm{d}}$ Department of Physics and Engineering, University of Zululand \\ P/B X 1041, KwaDlangezwa 3886, South Africa
}

\section{(Received December 14, 2016)}

Measurements of the proportional crosstalk were performed for the iThemba LABS segmented clover detector. More than 1000 crosstalk parameters were determined and a crosstalk correction algorithm was implemented successfully.

DOI:10.5506/APhysPolB.48.347

\section{Introduction}

Segmented detectors are new generation Ge detectors. They are able to measure the position of a $\gamma$-ray interaction inside the detector's volume. Their increased complexity results in a need for careful characterization. As a consequence of the segmentation on the outer contact, these detectors show proportional crosstalk that manifests itself as energy readings on the contacts that do not correspond to true energy deposition. To correct for proportional crosstalk dedicated measurements are needed.

The iThemba LABS segmented clover detector is of the TIGRESS [1] type. It comprises four Ge crystals, each of them segmented eight-fold on the outer contact, see Fig. 1. Thus, the detector has 32 signals from the segments and 4 from the inner cores.

* Presented by E.A. Lawrie at the Zakopane Conference on Nuclear Physics "Extremes of the Nuclear Landscape", Zakopane, Poland, August 28-September 4, 2016. 


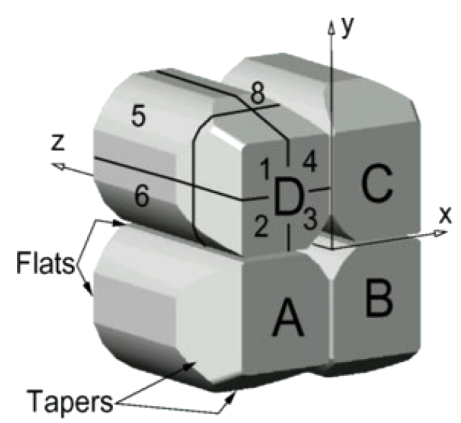

Fig. 1. A sketch showing the segmented clover, comprising four eight-fold segmented crystals.

When a $\gamma$ ray deposits its energy in a segment of the Ge crystal, the other segments register proportional crosstalk. It has the appearance of a charge collecting signal with small amplitude often with opposite polarity and generates an energy reading on the non-hit segments of the crystal, affecting the measurements that rely on segment data.

\section{Proportional crosstalk}

The magnitude of the crosstalk energy registered on a non-hit segment is proportional to the energy deposited in a hit segment. The crosstalk does not depend on the position of the interaction in the hit segment. It is specific for each pair of (hit, not-hit) contacts and for the segments, it is described by a $32 \times 32$ matrix, $\boldsymbol{B}$, of coefficients $\delta_{i j}$

$$
\begin{gathered}
\vec{E}_{\text {cross }}=\boldsymbol{B} \cdot \vec{E}_{\text {true }} \\
\boldsymbol{B}=\left(\begin{array}{ccccc}
1 & \delta_{1,2} & \delta_{1,3} & \ldots & \delta_{1, n} \\
\delta_{2,1} & 1 & \delta_{2,3} & \ldots & \delta_{1, n} \\
\delta_{3,1} & \delta_{3,2} & 1 & \ldots & \delta_{1, n} \\
\vdots & \vdots & \vdots & \ddots & \vdots \\
\delta_{n, 1} & \delta_{n, 2} & \delta_{n, 3} & \ldots & 1
\end{array}\right)
\end{gathered}
$$

In order to measure the crosstalk coefficients, three sources, ${ }^{152} \mathrm{Eu},{ }^{137} \mathrm{Cs}$ and ${ }^{60} \mathrm{Co}$, were placed in front of the detector. Data were collected using digital electronics [2] and digital data acquisition. An external trigger was built, such that data were recorded for all 36 channels if there was at least one Ge core signal larger than a set energy threshold. 
Data analysis was carried out in a similar way as in Ref. [3]. Events corresponding to a hit in a single segment in one crystal only were selected. The energies on the remaining 35 segments were measured as a function of the deposited energy in the hit segment. As an example, the measured crosstalk energies on the 7 non-hit segments of crystal A are shown in Fig. 2 as a function of the deposited energy in segment 5 of crystal A. The crosstalk energies show a linear dependence on the deposited energy. The slope of the curves shown in the figure is equal to the crosstalk coefficients $\delta_{5 j}$.

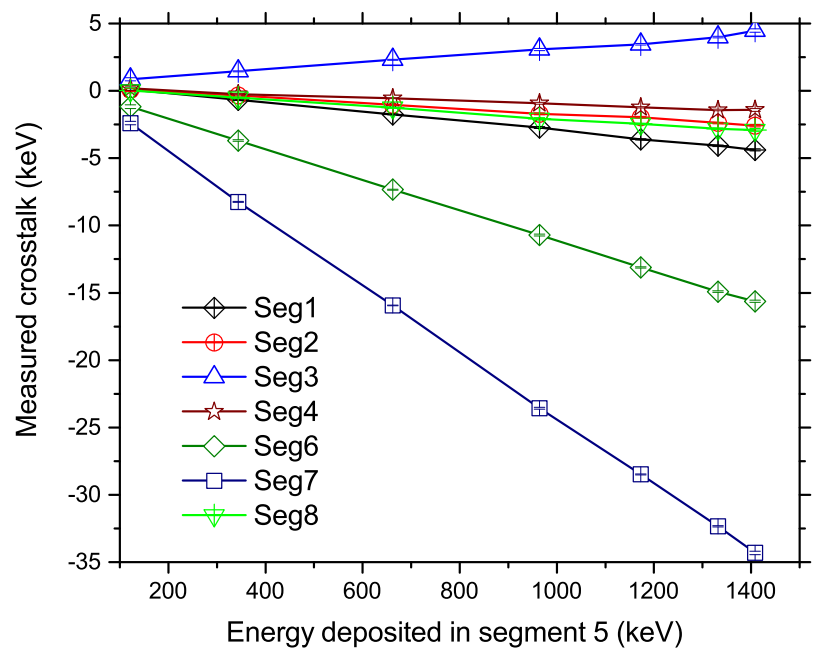

Fig. 2. Measured crosstalk on the non-hit segments of crystal A when segment 5 in crystal A was hit. The crosstalk energy is a linear function of the energy deposited in segment 5 .

The crosstalk coefficients extracted from the data were used to implement a crosstalk correction on the measured segment energies. As an example, we consider here all double-hit events in crystal A, where the hit segments were 5 and 7 . The addback spectrum, made as a sum of the energies registered in segments 5 and 7 of crystal A, is shown in the left panel of Fig. 3. The crosstalk created in segment 5 by the energy deposition in segment 7 distorts the measured energy in segment 5 . Similarly, the crosstalk created in segment 7 by the energy deposition in segment 5 distorts the measured energy in segment 7 . As a result, the addback spectrum in Fig. 3 (a) shows peaks that are widened at energy $E \lesssim 500 \mathrm{keV}$ and doubled at energy $E \gtrsim 1 \mathrm{MeV}$. Furthermore, the energy centroids of the peaks are shifted towards lower energies, yielding incorrect energy determination. To illustrate the crosstalk correction the same spectrum, but using the crosstalk corrected energies in segments 5 and 7 , is shown in Fig. 3 (b). The centroids of the peaks now appear at the correct energy. In addition, the peaks are single and narrow. 

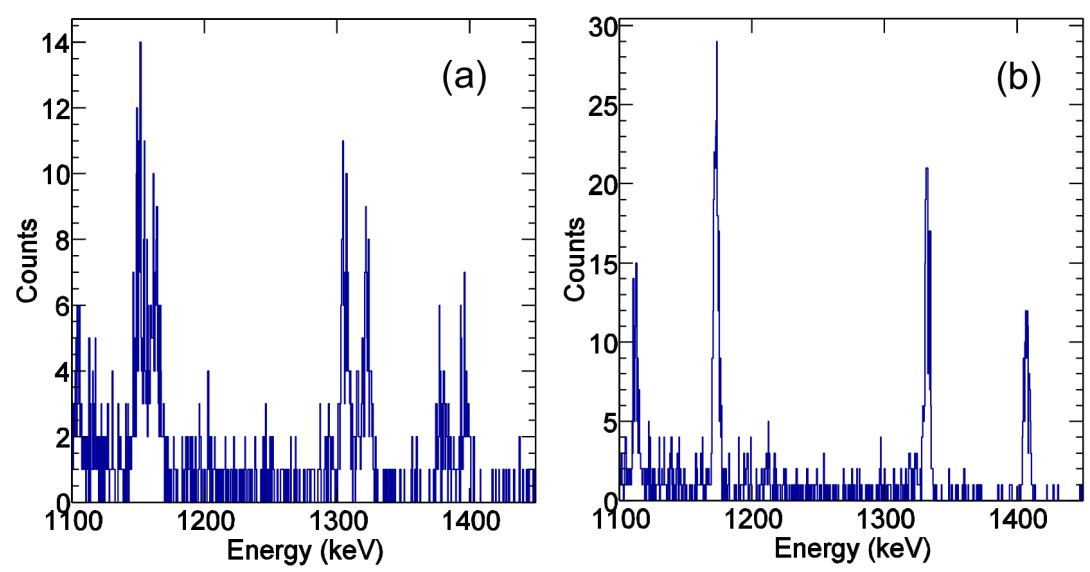

Fig. 3. (a) Addback spectrum generated as a sum of all double-hit events detected in segments 5 and 7 of crystal A. (b) The same as (a), but the addback spectrum is created after the detected energies are corrected for crosstalk.

\section{Summary}

The proportional crosstalk affects the registered energies on the segments of a segmented clover detector significantly. The crosstalk energy registered in a non-hit segment is a linear function of the energy deposited in a hit segment. The crosstalk coefficients were measured for the iThemba LABS segmented clover detector and an energy correction was successfully implemented. Such a correction is needed for any measurement that requires accurately determined segment energies.

This work is partially supported by the National Research Foundation, South Africa. The authors would like to thank the iThemba LABS technical, electronics, and IT staff for their excellent support. The assistance of Dr. P. Jones with the digital data acquisition is also greatly appreciated. This work benefited from many insightful discussions with Dr. B. Bruyneel, for which we thank him sincerely.

\section{REFERENCES}

[1] C.E. Svensson et al., Nucl. Instrum. Methods Phys. Res. A 540, 348 (2005).

[2] www.xia.com

[3] B. Bruyneel et al., Nucl. Instrum. Methods Phys. Rev. A 608, 99 (2009). 